\title{
Fast algorithm for radio propagation modeling in realistic 3-D urban environment
}

\author{
A. Rauch, J. Lianghai, A. Klein, and H. D. Schotten \\ University of Kaiserslautern, Paul-Ehrlich-Straße 11, 67663 Kaiserslautern, Germany \\ Correspondence to: J. Lianghai (ji@eit.uni-kl.de)
}

Received: 15 December 2014 - Revised: 6 April 2015 - Accepted: 26 April 2015 - Published: 3 November 2015

\begin{abstract}
Next generation wireless communication systems will consist of a large number of mobile or static terminals and should be able to fulfill multiple requirements depending on the current situation. Low latency and high packet success transmission rates should be mentioned in this context and can be summarized as ultra-reliable communications (URC). Especially for domains like mobile gaming, mobile video services but also for security relevant scenarios like traffic safety, traffic control systems and emergency management URC will be more and more required to guarantee a working communication between the terminals all the time.
\end{abstract}

\section{Introduction}

In order to evaluate the performance of next generation mobile communication systems in realistic deployment scenarios, system level simulation tools must be capable of integrating various models that reflect network deployment (e.g., antenna locations, elevation, orientation, transmit powers), user mobility and communication behavior, as well as service characteristics. The need for modeling system aspects and use cases of next generation mobile networks in a more realistic manner system is already acknowledged by European 5G research project ICT-317669 METIS (2013). In this paper we present a fast method for computing the path loss of micro and macro cells in a realistic, three dimensional urban environment scenario for moving and stationary users. Therefore, we use a simplified recursive ray tracing algorithm, which only takes into account one ray for each user per base station. Channel fading will be simulated by using Rayleigh and Rician distributed fading. To achieve a realistic Rician distributed fading, we use a variable $k$ factor that is randomly created taking into account the respective distance between transmitter and receiver. Both Rayleigh and Rician fading will be precomputed for the correspondent situations and just added to the path loss to achieve shorter computation times. Further, it is demonstrated that the employed algorithm is able to outperform the state-of-the-art approach described in Sect. 2.

The remainder of this paper is organized as follows: in Sect. 2 we give a short overview about the scenario layout and the user mobility. In Sect. 3 we present the path loss models and discuss the commonly used parameters. The computation of fast fading is presented in Sect. 4 while the simulation results are shown in Sect. 5.

\section{Urban environment and user mobility}

\subsection{3-D Scenario}

The three-dimensional scenario used in this paper is defined in ICT-317669 METIS (2013) and is called Madrid Grid scenario. This scenario consists of 15 buildings with different heights, one park area and corresponding streets between the buildings. The size of the scenario is 387 by $552 \mathrm{~m}$. An overview about the scenario is given in Fig. 1.

As shown in Fig. 1, there are 13 base stations considered namely 12 microcells (yellow) and one macrocell (red). The carrier frequency is $2.6 \mathrm{GHz}$ and $10 \mathrm{MHz}$ have been chosen as the system bandwidth.

Each road consists of two lanes for driving and two parking lanes. The road called "Gran Via" exhibits three lanes for driving in each direction has no parking lanes.

\subsection{User mobility}

The mobility model for vehicular user is adopted from ICT317669 METIS (2013). Here, every car moves witch the 


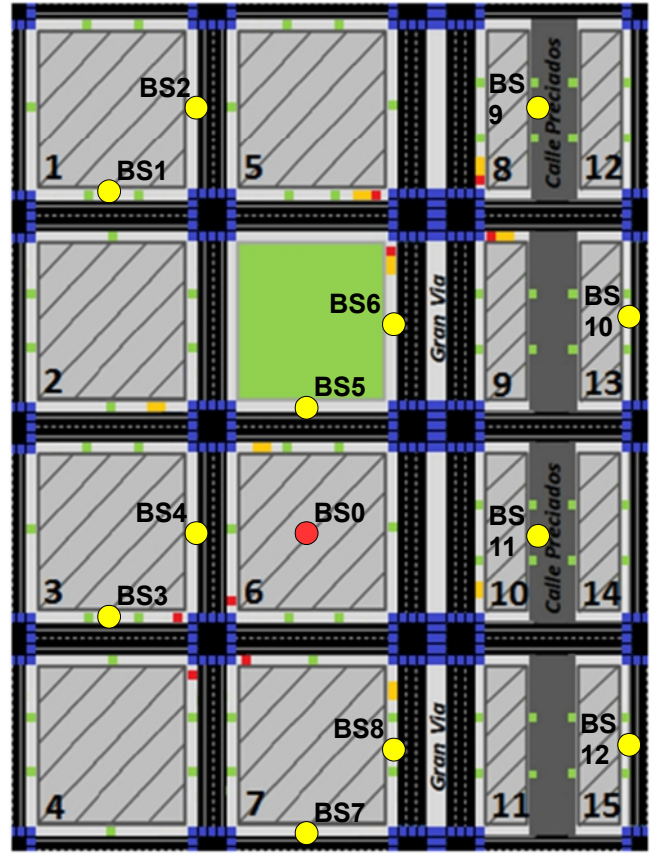

Figure 1. Madrid grid layout according to ICT-317669 METIS (2013).

same constant speed of $50 \mathrm{kmph}$. Changing directions is only possible at crossroads. The probability of a right or left turn is $25 \%$ respectively, while the probability of moving straight ahead is $50 \%$ at each crossroad.

\section{Path loss models}

\subsection{Computation of macrocell path loss}

In order to determine the path loss of macro cells, which are usually installed on rooftops, we use a path loss model that is proposed in ICT-317669 METIS (2013). This model takes into account the diffraction effects that radio signals experience and that is responsible for most of the signal energy received on ground level in urban environments. In this model, the calculation is divided in two parts. The first part $L_{\mathrm{fs}}$ describes the free space loss of signal from transmit antenna to the edge of the rooftop $\left(d_{\mathrm{r}}\right)$ and can be calculated as:

$L_{\mathrm{fs}}=-10 \log _{10}\left(\frac{\lambda}{4 \pi d_{\mathrm{r}}}\right)^{2}$.

The second part describes the diffraction loss of the signal from the rooftop to the receiver on street and is calculated as shown in Eq. (2).

$L_{\mathrm{rts}}=-10 \log _{10}\left[\frac{\lambda}{2 \pi^{2} r}\left(\frac{1}{\Theta}-\frac{1}{2 \pi+\Theta}\right)\right]$,

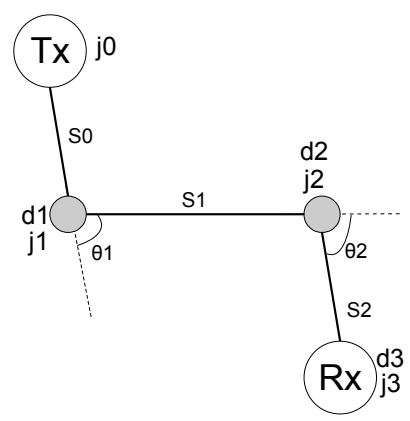

Figure 2. Street orientation according to Berg (1995).

where

$\Theta=\tan ^{-1}\left(\frac{\Delta h}{x}\right)$,

and

$r=\sqrt{(\Delta h)^{2}+x^{2}}$,

$\Delta h$ represents the difference of the building height and the mobile antenna height and $x$ the horizontal distance between the diffracting edge and the user. The resulting path loss is calculated as:

$L=L_{\mathrm{fs}}+L_{\mathrm{rts}}$.

Further details related to this model are shown in ICT317669 METIS (2013).

\subsection{Computation of microcell path loss}

In case of micro cells, which are installed below rooftop level in an urban environment, most of the signal energy reaches the user due to reflection between buildings, if the user is not in line of sight (LoS). Since tracing multiple signal reflections is computionally expensive, we choose two separate models to compute the overall microcell path loss.

The first model is used, if the user is in line of sight and the path loss is calculated as shown in is shown in Eq. (4).

$L_{\mathrm{dB}}=-10 \log _{10}\left(\frac{\lambda}{4 \pi d}\right),{ }^{2}$,

where $\lambda$ is the wavelength and $d$ is the distance between transmitter and receiver.

The second model is employed when the user is not in line of sight (nLoS). Here, we use Berg's recursive path loss model (Berg, 1995), which takes into account geometrical conditions, such as angle of crossroads and distance between two crossroads. In principle, an imaginary distance is calculated that depends on the real distances between transmitter and receiver and the angle between two streets.

As shown in Fig. 2, $s_{1}$ is the distance between crossing $j 1$ and $j 2 . s_{0}$ is the distance between transmitter $j 0$ and $j 1$, whereas $s_{2}$ is the distance between $j 2$ and receiver $j 3$. For 


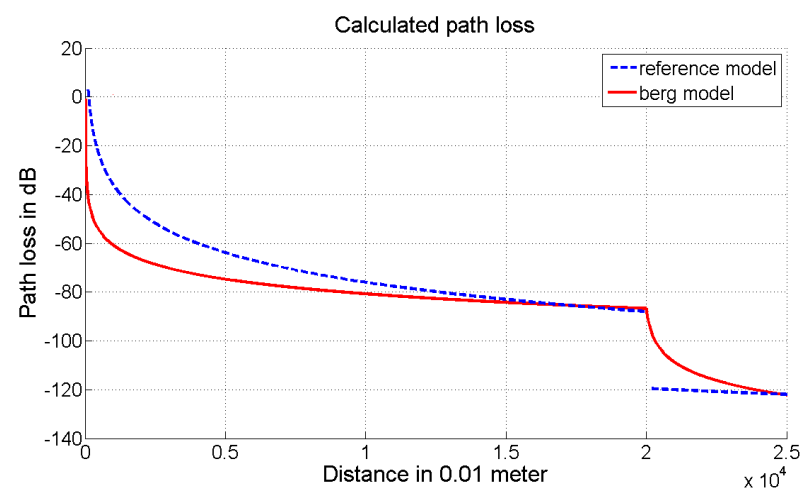

Figure 3. Calculated path loss for moving user.

the considered dense urban scenario angle $\Theta_{1}$ and $\Theta_{2}$ are set to $90^{\circ}$.

The imaginary distances are calculated according to Eq. (5) as described in Berg (1995).

$$
\begin{array}{r}
k_{j}=k_{j-1}+d_{j-1} q_{j-1}, \\
d_{j}=k_{j} s_{j-1}+d_{j-1},
\end{array}
$$

where $k_{0}=1$ and $d_{0}=0$. The variable $q$ represents the angle dependence of the path loss according to Eq. (6).

$q_{j}=\left(\Theta_{j} \frac{q_{90}}{90}\right)^{v}$.

In this case, $q_{90}$ is set to 0.5 and $v$ is set to 1.5 as recommended in Berg (1995). The path loss is now calculated as follows:

$L_{\mathrm{dB}}^{(n)}=-10 \log _{10}\left(\frac{\lambda}{4 \pi d_{n}}\right)^{2}$,

where $d_{n}$ is the imaginary distance between the respective transmitter and point $n$.

Figure 3 shows the corresponding path loss characteristic of a user moving with constant speed from point $\mathrm{P} 0$ to point P2 in Fig. 4. The dotted line shows the path loss of the reference model, which is further described in ICT-317669 METIS (2013) and ITU-R M.2135 (2009). The continuous line depicts the path loss of the bergs model, that is used in our approach. This approach leads to a smoother curve that may be adjusted by using the parameters $v$ and $q_{90}$ in Eq. (6).

The corresponding base station is depicted in yellow in Fig. 4 as well.

As illustrated in Fig. 4 the user is in line of sight of BS4 until he reaches P2. After turning left, the user is in $\mathrm{nLoS}$ and the corresponding path loss model for a $90^{\circ}$ crossroad is chosen.

Fading is not considered here.

\section{Computation of fast fading}

Simulating fast fading in detail according to the three dimensional scenario including multipath propagation due to

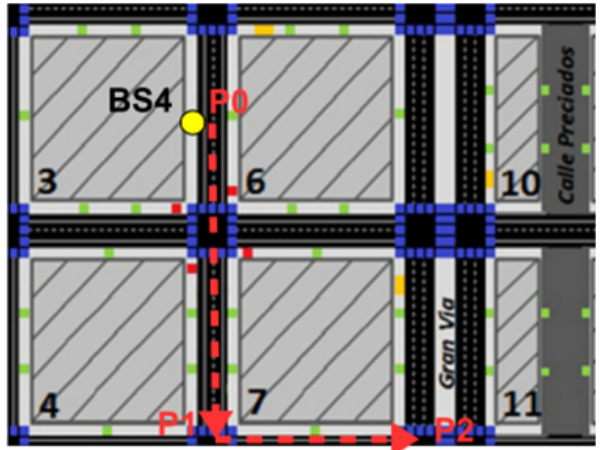

Figure 4. Movement of user.

reflection, diffraction and scattering would lead to a complex model that could not be computed in reasonable time. Since we simulate numerous moving users, the fast fading will be calculated by using some statistical approaches. As suggested in Tang and Hongbo (2003), Rayleigh and Rician distributions are used to generate fast fading. In the following sections, the utilization of both models is described.

\subsection{Rayleigh distributed fast fading}

If there is no dominant propagation path between transmitter and receiver (no line of sight) Rayleigh distributed random variables are used to describe fast fading, according to Eq. (8).

$P_{\text {Rayleigh }}(r)=\frac{r}{\sigma^{2}} e^{\frac{-r^{2}}{2 \sigma^{2}}}$,

where $\sigma^{2}$ represents the energy of the signal and $r$ the magnitude. To calculate the Doppler power spectral density, we use Eq. (9), which is proposed in Kostov (2003), for the Rayleigh and Rician channel:

$$
S(f)=\frac{1}{\pi f_{m} \sqrt{1-\left(\frac{f}{f_{m}}\right)^{2}}},
$$

where $f_{m}$ is the Doppler frequency shift that is calculated as:

$f_{m}=\frac{v f_{\mathrm{c}}}{c}$,

$v=50 \mathrm{kmph}$ represents the user speed, $f_{\mathrm{c}}$ is the carrier frequency, which is $2.6 \mathrm{GHz}$ in this case and $c=3 \times 10^{8} \mathrm{~m} \mathrm{~s}^{-1}$ is the speed of light. The Rayleigh fading will be precalculated, stored and simply added to the path loss during the simulation.

\subsection{Rician distributed fast fading}

To simulate multipath propagation where at least one path is much stronger than the others (line of sight), we employ 


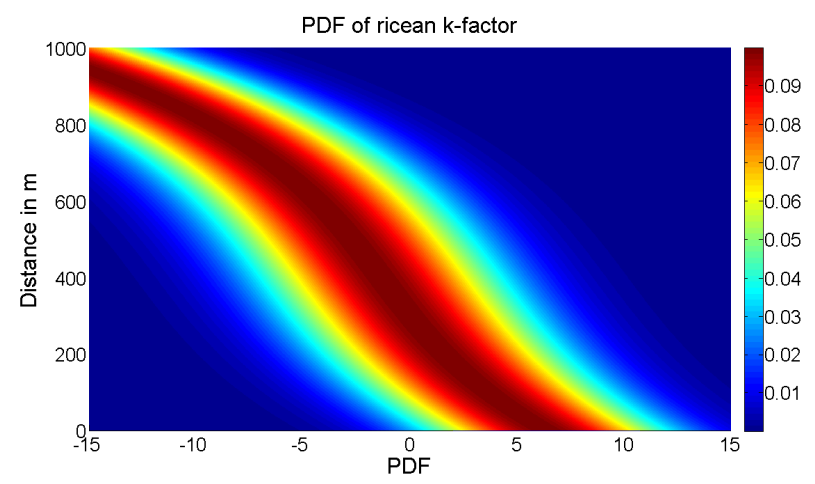

Figure 5. PDF of the ricean $k$ factor.

Rician distributed random variables. Rician distributed fast fading can be described as:

$P_{\text {Rice }}(r)=\frac{r}{\sigma^{2}} e^{-\frac{r^{2}}{2 \sigma^{2}}+K} I_{0}\left[\frac{r \beta}{\sigma^{2}}\right], \forall r \geq 0$

with $K=\frac{\beta^{2}}{2 \sigma^{2}}$ (Jeruchim et al., 2000; Iskander, 2008).

For $K=0$ and $I_{0}\left[\frac{r \beta}{\sigma^{2}}\right]=1$ Eq. (11) leads again to the Rayleigh fading calculated in Eq. (8) as proposed in Kostov (2003). The variable $K$ is called Ricean $k$ factor and rather describes the relation between the signal of the line of sight path and the non line of sight paths. As proposed in Thiele et al. (2006), the non line of sight signal components need to be summed up to determine the $k$ factor. To avoid simulating every single signal path we use the statistical approaches to estimate the $k$ factor, that are described in Thiele et al. (2006), Medawar et al. (2013) and Greenstein et al. (1999). In Thiele et al. (2006) and Medawar et al. (2013) functions are provided that take into account the urban environment and the distance between transmitter and receiver resulting in a cumulative distribution function (CDF) of the Rician $k$ factor.

The $k$ factor is now calculated using the line of sight distance from transmitter to receiver and a random value, which is generated according to the corresponding probability distribution function. Figure 5 shows the probability distribution function (PDF) of the $k$ factor over the distance, where the $y$ axis represents the distance in meters, the $z$ axis stands for the probability, and the $x$ axis shows the $k$ factor in $\mathrm{dB}$.

As shown in Fig. 5, the probability of a high $k$ factor value decreases with an increasing distance between receiver and transmitter. In our simulation tool the $k$ factor values are stored separately and used to generate Rician fading for the relevant distance between transmitter and receiver according to Eq. (11) (Jeruchim et al., 2000; Iskander, 2008).

Figures 6 and 7 show the fast fading for a $k$ factor of 0 and $15 \mathrm{~dB}$ respectively.

For $k=0$ the Ricean fading transfers into Rayleigh fading as shown in Eqs. (11) and (8).

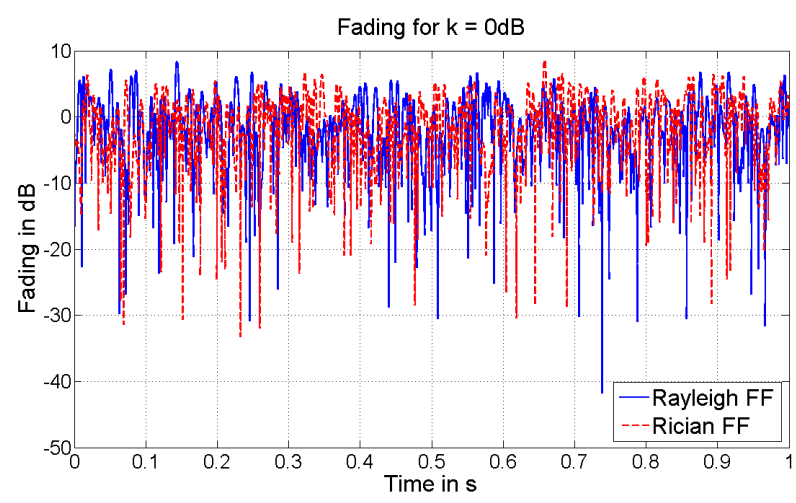

Figure 6. Ricean fading for $k=0 \mathrm{~dB}$.

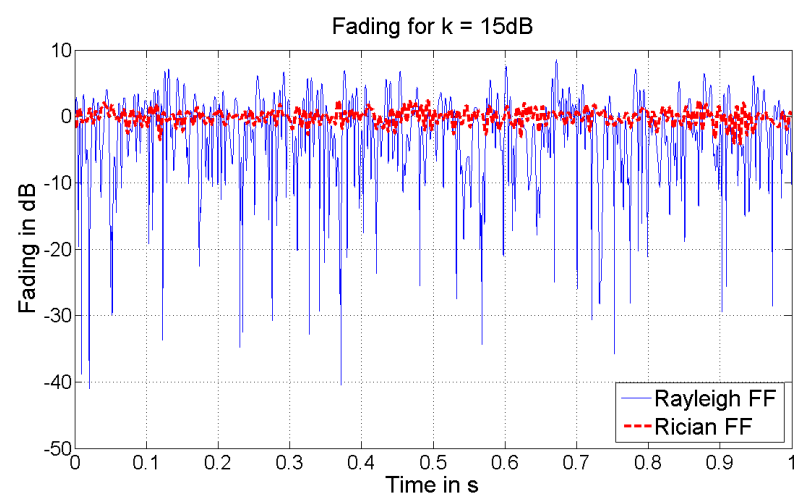

Figure 7. Ricean fading for $k=15 \mathrm{~dB}$.

\section{Simulation results}

In this section, we will present some simulation results regarding the path loss of a moving user in the described scenario. Figure 8 shows the path loss between transmitter and a moving user that follows the same route as described in Fig. 4. The Rician $k$ factor for $\mathrm{nLoS}$ conditions changes due to the distance as shown in Fig. 5. The appropriate models for fading and propagation will be chosen automatically using a single ray to detect NLoS or LoS conditions. The simulated path loss from P0 to P2 is shown in Fig. 8.

Since the main objective of this approach was to find a method that is fast enough to simulate numerous randomly distributed user in a realistic, 3-D-environment, we compare this method to a reference method, that is further described in ICT-317669 METIS (2013) and ITU-R M.2135 (2009). This method does not precompute the fading and does not use the recursive method to estimate the path loss. Further the computation of numerous log-operations is necessary here, which takes some computing time. In Fig. 9 we compare the averaged frame refresh rate during a simulation set over an increasing number of simulated users in the same scenario and the same user positions. 


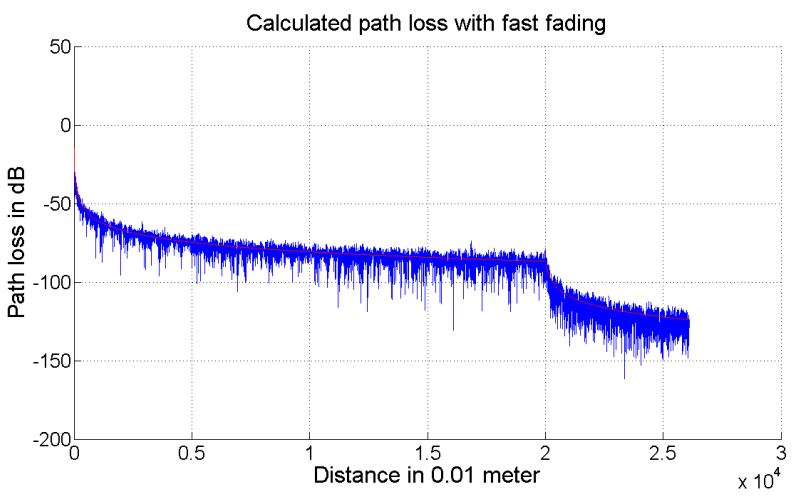

Figure 8. Path loss with fast fading.

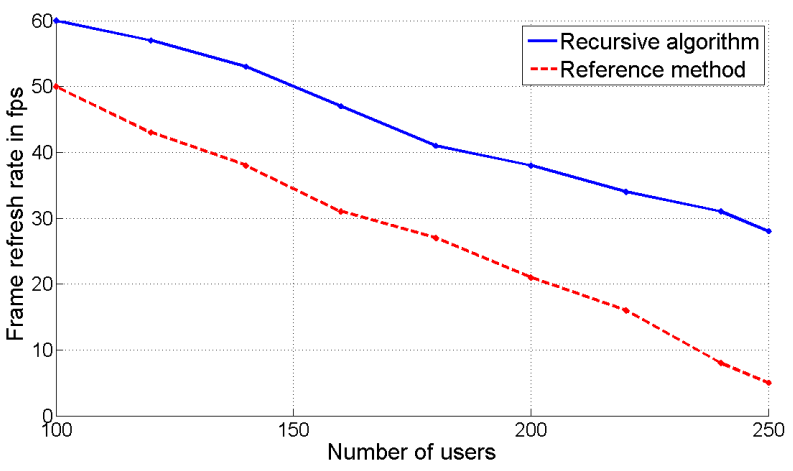

Figure 9. Refresh rate vs. number of users.

As illustrated in Fig. 9 we can simulate up to 80 more users with the method proposed in this paper at a frame refresh rate of $30 \mathrm{fps}$ than with the reference method.

\section{Conclusions}

In this work, we use a simplified model for efficiently computing the path loss between receiver and transmitter in line of sight conditions as well as in non line of sight conditions using Berg's recursive model. To achieve realistic simulation results we combine this model with fast fading models, such as Rayleigh distributed fading for non line of sight propagation. To consider multipath fading in line of sight conditions, we employ Rician distributed fading with a variable $k$ factor, which depends on the distance between transmitter and receiver. The proposed model was compared with a reference model that does not use pre-computation and recursive algorithms. The model presented in this paper leads to a better performance that allows to simulate more moving users in a realistic 3-D-environment.
Acknowledgements. Part of this work has been performed in the framework of the FP7 project ICT-317669 METIS - Mobile and wireless communications Enablers for the 2020 Information Society, which is partly funded by the European Commission. This paper expresses the authors views, which are not necessarily those of the METIS consortium. The authors alone are responsible for the content of this paper.

Edited by: M. Chandra

Reviewed by: two anonymous referees

\section{References}

Berg, J.-E.: A Recursive Method For Street Microcell Path Loss Calculation, Proc. IEEE International Symposium on Personal, Indoor and Mobile Radio Communications, 1, 140-143, 1995.

Greenstein, L., Michelson, D., and Erceg, V.: Moment-Method Estimation of the Rician K-Factor, IEEE Comm. Lett., 3, 175-176, doi:10.1109/4234.769521, 1999.

ICT-317669 METIS: Deliverable 6.1, ICT-317669, available at https://www.metis2020.com, 2013.

Iskander, C.-D.: A MATLAB-based Object-Oriented Approach to Multipath Fading Channel Simulation, white paper, February 2008.

ITU-R M.2135: Guidelines for evaluation of radio interface technologies for IMT-Advanced, M.2135, available at: https://www.itu.int/dms_pub/itu-r/opb/rep/R-REP-M. 2135-1-2009-PDF-E.pdf, 2009.

Jeruchim, M. C., Balaban, P., and Shanmugan, K. S.: Simulation of Communication Systems, 2nd Edn., New York, Kluwer Academic/Plenum, 2000.

Kostov, N.: Mobile Radio Channels Modeling in MATLAB, Department of Radio Engineering, Technical University of Varna, RadioEngineering, 12, 12-16, 2003.

Medawar, S., Haendel, P., and Zetterberg, P: Approximate Maximum Likelihood Estimation of Rician K-Factor and Investigation of Urban Wireless Measurements, Trans. Wireless Comm., 12, 2545-2555, doi:10.1109/TWC.2013.042413.111734, 2013.

Saunders, S. R.: Antennas and propagation for wireless communication systems, John Wily \& Sons, Ltd., New York, 1999.

Tang, L. and Hongbo, Z.: Analysis and Simulation of Nakagami Fading Channel with MATLAB, Asia-Pacific Conference on Environmental Electromagnetics Hangzhou, 2003.

Thiele, L., Peter, M., and Jungnickel, V.: Statistics of the rician kfactor at $5.2 \mathrm{GHz}$ in an urban macro-cell scenario, IEEE International Symposium on Personal, Indoor and Mobile Radio Communications, 1, 2006. 